\title{
Multi-Source Multi-Sink Stochastic-Flow Networks Reliability under Time Constraints
}

\author{
M. R. Hassan ${ }^{1}$ and H. Abdou' ${ }^{1,2}$ \\ 'Department of Mathematics, Computer Science Branch, Mathematics Department, Aswan University, Aswan, \\ Egypt; m_r_hassan73@yahoo.com \\ ²Department Applied Natural Sciences, Qassim University, Buraydah, Saudi Arabia; haniabdou2000@yahoo.com
}

\begin{abstract}
Objectives: This study is centered on four issues related to the reliability evaluation in multi-source multi-sink networks. Each issue discusses the reliability evaluation under different condition. These conditions play an important role in determining the quickest paths used in transmitting data between source and sink nodes, with the condition that the transmission time of the quickest path does not surpass a predetermined upper bound T. Methods/Statistical Analysis:

Proposed algorithms used in each issue designed based on approaches taken from previous literatures to evaluate the reliability. Findings: The reliability $\left(R_{d_{w j} T}\right.$ ) evaluated when each source transmits the demand $\mathrm{d}_{\mathrm{wj}}$ (the demand for resource $w$ at sink node $\mathrm{t}_{\mathrm{j}}$ ) separately to the distinct sink, this is the first issue. The second issue deals with transmitting demands request by sink nodes from one source via a group of disjoint paths. In the case of transmitting demands through a gathering of joint paths, share one or more common arc, this is the third one. The last issue investigates the problem of sending demands requested by all sinks from all sources via joint paths. Application/Improvements: Examples are presented to illustrate how to evaluate the reliability of a multi-source multi-sink networking each case under time constraint.
\end{abstract}

Keywords: Joint and Disjoint Paths, Multi-Source Multi-Sink Stochastic-Flow Networks, Time Constraint, Quickest Path, System Reliability

\section{Introduction}

The quickest path problem is to obtain a routing path in a network with a minimum time to ship $\sigma$ units of data from the source to the $\sin ^{1}{ }^{1}$. $\mathrm{In}^{2}$, the proposed method is targeted towards the situation where multi-commodities are conveyed through all disjointed minimal paths (MPs) in a network. In ${ }^{3}$, distributed algorithms are developed for the quickest path problem in any a synchronous communications networks. In ${ }^{4}$ the problem is supposed as a criteria path problem, allowing the use of a very efficient algorithm, which solves the quietest path problem for all possible values of the amount of data that has to be transmitted.
The system reliability of stochastic-flow networks under time constraint is defined as the probability of sending $d$ units of data from the source to the sink through the network within $T$ units of time, denoted by $\mathrm{R}_{\mathrm{d}, \mathrm{T}}{ }^{5-7}$. The problem of determining the optimal routing policy with the highest system reliability discussed in $^{8}$ and ${ }^{9}$. Network reliability has been evaluated in the case of sending units of data through a number of MPs simultaneously under both time and budget constraints ${ }^{10}$. Moreover, network reliability according to the spare routing was evaluated ${ }^{11}$. In order to reduce transmission time, the problem of simultaneously transmitting data through multiple disjoint minimal paths was presented in ${ }^{12}$.

*Author for correspondence 
A multi-source multi-sink stochastic-flow network is an extension of the concept to multiple sources and sinks on the same network. Evaluating the system reliability of multi-source multi-sink stochastic flow networks has furthermore been addressed in $\frac{13-16}{}$. In $\frac{13}{3}$, the optimal resource allocation problem subject to reliability maximization has been formulated and presented an algorithm to solve it. For more than one resource ${ }^{14}$, the optimal resource flow allocation problem has been studied and a GA was proposed to solve it. In $\frac{15}{}$, the flow allocation problem subject to transportation cost was studied and solved using GAs. $\mathrm{I} \mathrm{n}^{\frac{16}{6}}$, the author modified and solved the formulation of the flow allocation problem subject to the probability of the capacity vector and transmission cost. Further, system reliability was evaluated by searching for the optimal lower boundary points

In this paper, we will extend the quickest path problem to multi-source multi-sink flow networks. The presented problem has been studied under the following cases:

1. Each source node $\left(s_{i}\right)$ sends the specified demand $d_{w j}$ (demand for resource $w$ at sink node $t_{j}$ ) separately to each sink $\left(t_{j}\right)$.

2. Each source node $\left(s_{\mathrm{i}}\right)$ sends the specified demands to all sink nodes $\left(t_{i}, j=1,2, \ldots\right.$, through different paths that do not share any common arcs (disjoint paths).

3. Each source node $\left(s_{i}\right)$ sends the specified demands to all sink nodes $\left(t_{j}, j=1,2, \ldots\right.$, through joint paths that share some arcs.

4. All source nodes $\left(s_{i}, i=1,2,3, \ldots\right)$ sends multiple demands to all sink nodes $(t, j=1,2, \ldots$,$) through joint$ paths, the general case, simultaneously transmitting.

The rest of the paper is organized as follows. Section 2 presents notations and assumptions. Section 3 presents Case A: transmitting demands separately. Section 4 describes Case B: transmitting demands through disjoint paths. Section 5 provides Case C: transmitting demands through joint paths. Case D: the general case, when transmitting multiple demands from all sources to all sinks given in Section 6. Section 7 offers our conclusions.

\section{Notation and Assumptions}

\subsection{Notation}

$G(A, N, M, S, T)$ a multi-source multi-sink stochasticflow network.
A $\left\{\mathrm{a}_{\mathrm{e}} \mid 1 \leq \mathrm{e} \leq \mathrm{n}\right\}$, set of arcs.

$\mathrm{N}$ set of nodes.

M $M=\left\{M_{1}, M_{2}, \ldots, M_{n}\right\}$, where $M_{e}$ is the maximum capacity of each arc $a_{e}$.

S $\quad\left\{s_{1}, \ldots, s_{\mathrm{q}}\right\}$ : set of source nodes.

$\mathrm{T} \quad\left\{\mathrm{t}_{1}, \ldots, \mathrm{t}_{\theta}\right\}$ : set of sink nodes.

$\mathrm{D}\left\{\mathrm{d}_{\mathrm{w}, \mathrm{j}} \mid 1 \leq w \leq \mathrm{m}, 1 \leq \mathrm{j} \leq \theta\right\}$, where $\mathrm{d}_{w, \mathrm{j}}$ is the demand for resource $w$ at sink node $t_{j}$.

MP Minimal path,

$\mathrm{MP}_{\mathrm{i}, \mathrm{j}, \mathrm{k}}$ The $\mathrm{k}^{\text {th }}$ MP from $\mathrm{s}_{\mathrm{i}}$ to $\mathrm{t}_{\mathrm{j}}$.

MPS $\left\{M P_{i, j, k} \mid 1 \leq i \leq \sigma, 1 \leq j \leq \theta, 1 \leq k \leq k_{i, j}\right\} \quad:$ a

set of all MPs, where $\mathrm{k}_{\mathrm{i}, \mathrm{j}}$ represents the number of MPs from $s_{i}$ to $t_{j}$.

$\mathrm{L}_{\mathrm{i}} \quad$ The lead time of arc

$\mathrm{L}\left(\mathrm{MP}_{\mathrm{ijk}}\right)$ The total lead time of the path $\left(\mathrm{MP}_{\mathrm{ijk}}\right)$

$W^{w j}\left(a_{l}\right)$ is the consumed capacity of by commodity $\mathrm{d}_{\mathrm{wj}}$.

np Total number of MPs contained in MPS.

$\mathrm{X}$ Capacity vector defined as $\mathrm{X}=\left(x_{1}, x_{2}, \ldots x_{e}, \ldots, x_{n}\right)$. $R_{d_{j i}, T}$ The system reliability for the given demand $\mathrm{d}_{\mathrm{wj}}$ under $\mathrm{T}$.

\subsection{Assumptions}

1. The capacity of each arc $a_{e}$ is an integer-valued random variable, which takes values $0<1<2<\ldots<\mathrm{M}_{\mathrm{e}}$ according to a given distribution.

2. The capacities of the arcs are statistically independent.

3. The flow along a path does not exceed its maximum capacity.

\section{Case A: Transmitting Demands Separately from One Source to One Sink}

The following subsections describe how to calculate i.e. the reliability of transmitting a single demand from the source node to the sink. 


\subsection{Definition of Lower Boundary Points for $\left(\mathrm{d}_{\mathrm{wj}}, \mathrm{T}\right)$}

If $\mathrm{X}$ is a minimal capacity vector such that the network can send $d_{w j}$ units of data from the source to the sink within $\mathrm{T}$ units of time, then $\mathrm{X}$ is called a lower boundary point for $\left(\mathrm{d}_{\mathrm{wj}}, \mathrm{T}\right)$.

\subsection{Generate All Lower Boundary Points for $\left(d_{w j}, T\right)$.}

In the following steps, for the $\mathrm{k}^{\text {th }} \mathrm{MP}, \mathrm{MP}_{\mathrm{i}, \mathrm{j}, \mathrm{k}}$ from $\mathrm{s}_{\mathrm{i}}$ to $\mathrm{t}_{\mathrm{j},} \mathrm{MP}_{\mathrm{i}, \mathrm{j}, \mathrm{k}}=\{,, \ldots$,$\} , we will show how to find the mini-$ mal capacity vector $X^{i, j}=\left(x_{1}, x_{2}, \ldots, x_{e}, \ldots, x_{n}\right)$ such that the network sends $\mathrm{d}_{\mathrm{wj}}$ units of data within T units of time from the source $s_{i}$ to the sink $t_{j}$.

1. For each $\mathrm{MP}_{\mathrm{ijk}}$, determine the smallest integer $v$ such that,

$$
\sum_{i=1}^{n}\left\{1_{\mathrm{i}} \mid a_{i} \in \boldsymbol{m}{ }_{j, j, k}\right\}+\left\lceil d_{j} / v\right\rceil \leq T
$$

2. If , generate the system capacity vector $\mathrm{X}^{\mathrm{i}, \mathrm{j}}=\left(x_{l^{\prime}}\right.$ $\left.x_{2}, \ldots, x_{2}, \ldots, x_{n}\right)$ for each $\mathrm{MP}_{\mathrm{i}, \mathrm{j}, \mathrm{k}}$ as follows:

$$
x_{e}=\left\{\begin{array}{cc}
u \geq v & \text { if } a_{i} \in M P_{i, j, k} \\
0 & \text { otherwise }
\end{array}\right.
$$

Where $\mathrm{x}_{\mathrm{e}}$ is an element of $\mathrm{X}^{\mathrm{i}, \mathrm{j}}$ and $u$ is the minimal capacity of $a_{i}$.

\subsection{Evaluation of $R_{d_{\dot{w}}, T}$}

If $\mathrm{X}_{1}^{\mathrm{i}, \mathrm{j}}, \mathrm{X}_{2}^{\mathrm{i}, \mathrm{j}}, \ldots, \mathrm{X}_{\mathrm{q}}^{\mathrm{i}, \mathrm{j}}$ are the collection of all $\left(\mathrm{d}_{\mathrm{wj}}, \mathrm{T}\right)-\mathrm{MP}_{\mathrm{i}, \mathrm{j}}$, and then the system reliability $R_{d_{j} \text { T }}$ is defined as follows:

$$
R_{\left(d_{\dot{w}}, T\right)}=\operatorname{Pr}\left\{\bigcup_{u=1}^{q}\left\{Y \mid Y \geq X_{u}^{i, j}\right\}\right.
$$

Where $\operatorname{Pr}\{\mathrm{Y}\}=\operatorname{Pr}\left\{\mathrm{y}_{1}\right\} \cdot \operatorname{Pr}\left\{\mathrm{y}_{2}\right\} \cdot \ldots \cdot \operatorname{Pr}\left\{\mathrm{y}_{\mathrm{n}}\right\}$.

Several methods ${ }^{16-20}$ can be used to evaluate (3), in this paper, we will use to evaluate $R_{d_{\dot{w}} T}$.

\subsection{Illustrative Example}

As an example, we consider the network in Figure 1, which has two source and two sink nodes. The arcs are numbered from $\mathrm{a}_{1}$ to $\mathrm{a}_{14}$; their capacities, corresponding probabilities and lead-time of each arc taken from ${ }^{15,16}$.

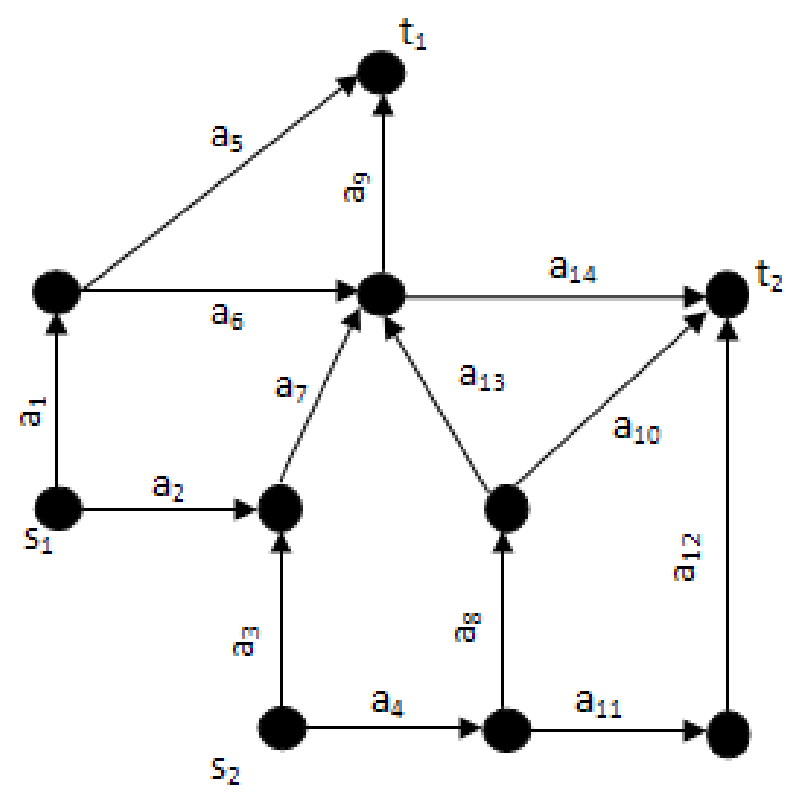

Figure 1. Two-source two-sink computer network.

In the following steps, we will show how to calculate $R_{d 11, T}$, i.e., the reliability from the source node $s_{1}$ to the sink node $t_{1}$, where $d_{11}=11$ and $T=9$, i.e. evaluate $R_{11,9}$. There are three MPs from $\mathrm{s}_{1}$ to $\mathrm{t}_{1}: M P_{1,1,1}=\left\{a_{1}, a_{5}\right\}, M P_{1,1,2}$ $=\left\{a_{1}, a_{6}, a_{9}\right\}, M P_{1,1,3}=\left\{a_{2}, a_{7}, a_{9}\right\}$.

Step 1: (1.1) the lead-time of $\mathrm{MP}_{1,1,1}=\left\{\mathrm{a}_{1}, \mathrm{a}_{5}\right\}$ is $l_{1}+l_{5}=3$. Then $\mathrm{v}=2$ is the smallest integer such that $\left(3+\left\lceil\frac{1}{2}\right\rceil\right) \leq 9$.

(1.2) the maximal capacity of $\mathrm{MP}_{1,1,1}$ is 8 . Hence, $x_{1}=x_{5}=2$ and $x_{i}=0$ for others. So we obtain $X_{1}$ $=(2,0,0,0,2,0,0,0,0,0,0,0,0,0)$.

Step 2: (1.1) the lead-time of $M P_{1,1,2}=\left\{a_{1}, a_{6}, a_{9}\right\}$ is $l_{1}+l_{6}+l_{9}=7$. Then $\mathrm{v}=6$ is the smallest integer

Such that $\left(7+\left\lceil\frac{1}{6}\right\rceil\right) \leq 9$.

(1.2) the maximal capacity of $\mathrm{MP}_{1,1,2}$ is 10 . Hence, $x_{1}+x_{6}+x_{9}=6$ and $x_{i}=0$ for others. So we obtain $X_{2}$ $=(6,0,0,0,0,6,0,0,6,0,0,0,0,0)$. 
Step 3: (1.1) the lead-time of $M P_{1,1,3}=\left\{a_{2}, a_{7}, a_{9}\right\}$ is $l_{2}+l_{7}+l_{9}=6$. Then $\mathrm{v}=4$ is the smallest integer such that $\left(6+\left\lceil\frac{1}{4}\right\rceil\right) \leq 9$.

\begin{tabular}{|l|l|l|l|l|l|}
\hline $\mathrm{a}_{3}$ & 1 & 2 & $\mathrm{a}_{10}$ & 1 & 2 \\
\hline $\mathrm{a}_{4}$ & 1 & 2 & $\mathrm{a}_{11}$ & 1 & 2 \\
\hline $\mathrm{a}_{5}$ & 1 & 2 & $\mathrm{a}_{12}$ & 1 & 2 \\
\hline $\mathrm{a}_{6}$ & 1 & 2 & $\mathrm{a}_{13}$ & 1 & 2 \\
\hline $\mathrm{a}_{7}$ & 1 & 2 & $\mathrm{a}_{14}$ & 1 & 2 \\
\hline
\end{tabular}

(1.2) the maximal capacity of $\mathrm{MP}_{1,1,3}$ is 10 . Hence, $x_{2}+x_{7}+x_{9}=4$ and $x_{i}=0$ for others. So we obtain $X_{3}$ $=(0,4,0,0,0,0,4,0,4,0,0,0,0,0)$.

Hence $\mathrm{R}_{11,9}=0.999957$ using Eq (3). Table 1 summarizes the values of $R_{d_{j i}, T}$ for different values of $\mathrm{d}_{\mathrm{wj}}$.

Table 1. Values of $R_{d_{w j}, T}$ for different values of $\mathrm{d}_{\mathrm{wj}}$

\begin{tabular}{|l|l|l|l|}
\hline$\left(\mathbf{s}_{\mathbf{i}}, \mathbf{t}_{\mathbf{j}}\right)$ & $\mathbf{M P}_{\mathrm{i}, \mathrm{j}, \mathbf{k}}$ & $R_{d_{w j}, T}$ & $\begin{array}{l}\text { The value of } \\
R_{d_{w j}, T}\end{array}$ \\
\hline$(1,2)$ & $\begin{array}{l}\mathrm{MP}_{1,2,1}=\left\{\mathrm{a}_{1}, \mathrm{a}_{6}, \mathrm{a}_{14}\right\} \\
\mathrm{MP}_{1,2,2}=\left\{\mathrm{a}_{2}, \mathrm{a}_{7}, \mathrm{a}_{14}\right.\end{array}$ & $R_{12,9}$ & 0.995772 \\
\hline$(2,1)$ & $\begin{array}{l}\mathrm{MP}_{2,1,1}=\left\{\mathrm{a}_{2}, \mathrm{a}_{7}, \mathrm{a}_{9}\right\} \\
\mathrm{MP}_{2,1,2}=\left\{\mathrm{a}_{4}, \mathrm{a}_{8}, \mathrm{a}_{9}, \mathrm{a}_{13}\right\}\end{array}$ & $R_{7,9}$ & 0.993354 \\
\hline$(2,2)$ & $\begin{array}{l}\mathrm{MP}_{2,2,1}=\left\{\mathrm{a}_{3}, \mathrm{a}_{7}, \mathrm{a}_{14}\right\} \\
\mathrm{MP}_{2,2,2}=\left\{\mathrm{a}_{4}, \mathrm{a}_{8}, \mathrm{a}_{13}, \mathrm{a}_{14}\right\} \\
\mathrm{MP}_{2,2,3}=\left\{\mathrm{a}_{4}, \mathrm{a}_{8}, \mathrm{a}_{10}\right\} \\
\mathrm{MP}_{2,2,4}=\left\{\mathrm{a}_{4}, \mathrm{a}_{1}, \mathrm{a}_{12}\right\}\end{array}$ & $R_{10,9}$ & 0.999881 \\
\hline
\end{tabular}

\section{Case B: Transmitting Demands through Disjoint Paths}

We study how to calculates $R_{D_{w j}^{2}}$, , the reliability from the source node $s_{1}$ to the sink nodes $t_{1}$ and $t_{2}$,where $D_{w j}^{u}=\left(d_{11}, d_{12}\right)$. There are five MPs from $\mathrm{s}_{1}$ to both $\mathrm{t}_{1}$ and $\mathrm{t}_{2}$ shown in Table 2-3. In this case, the following constraint for each bandwidth should be satisfied when is sent through.

$$
b_{k}^{w j} \leq B_{k}
$$

Table 2. The consumed capacity

\begin{tabular}{|l|l|l|l|l|l|}
\hline $\operatorname{Arc}$ & $\mathrm{W}^{11}\left(\mathrm{a}_{1}\right)$ & $\mathrm{W}^{12}\left(\mathrm{a}_{1}\right)$ & $\operatorname{Arc}$ & $\mathrm{W}^{11}\left(\mathrm{a}_{1}\right)$ & $\mathrm{W}^{12}\left(\mathrm{a}_{1}\right)$ \\
\hline $\mathrm{a}_{1}$ & 1 & 2 & $\mathrm{a}_{8}$ & 1 & 2 \\
\hline $\mathrm{a}_{2}$ & 1 & 2 & $\mathrm{a}_{9}$ & 1 & 2 \\
\hline
\end{tabular}

Table 3. The data of each MP and $B_{k}$ values

\begin{tabular}{|l|l|l|l|}
\hline $\mathbf{k}$ & $\mathbf{M P}_{\mathrm{ijk}_{\mathrm{k}}}$ & $\mathrm{L}\left(\mathrm{MP}_{\mathrm{ijk}}\right)$ & $\mathrm{B}_{\mathbf{k}}$ \\
\hline 1 & $\left\{\mathrm{a}_{1}, \mathrm{a}_{5}\right\}$ & 3 & 8 \\
\hline 2 & $\left\{\mathrm{a}_{1}, \mathrm{a}_{6}, \mathrm{a}_{9}\right\}$ & 7 & 10 \\
\hline 3 & $\left\{\mathrm{a}_{2}, \mathrm{a}_{7}, \mathrm{a}_{9}\right\}$ & 6 & 10 \\
\hline 4 & $\left\{\mathrm{a}_{1}, \mathrm{a}_{6}, \mathrm{a}_{14}\right\}$ & 6 & 10 \\
\hline 5 & $\left\{\mathrm{a}_{2}, \mathrm{a}_{7}, \mathrm{a}_{14}\right\}$ & 5 & 10 \\
\hline
\end{tabular}

Where is the upper bound for bandwidth of, it is given by:

$$
B_{k}=\min _{a_{i \in M P_{i j k}}}\left\{c\left(a_{l}\right)\right\}
$$

The $b_{k}^{w j}$ bandwidth is evaluated by the following equation:

$$
b_{k}^{w j}=\frac{\max _{a_{l} \in M P_{i j k}}\left\{W^{w j}\left(a_{l}\right)\right\} \times d_{w j}}{T-L\left(M P_{i j k}\right)}
$$

Finally, each in the capacity vector $\mathrm{X}, \mathrm{X}=()$, is constructed by eq. (7), (2).

$x_{h}=\sum_{k=1}^{n p} \sum_{w, j=1,1}^{m, \theta} \rho_{k} b_{k}^{w j}$, where $\rho_{k} \begin{cases}1, & \text { if } a_{h} \in M P_{i j k} \\ 0 & \text { if } a_{h} \notin M P_{i j k}\end{cases}$

The following algorithm is used to evaluate the reliability for Case B.

\section{Algorithm B \\ Begin}

B.1. For each arc; Read $\mathrm{p}$ (, and.

B.2. Determine the source node and the sink nodes .

B.3. Read, T, and and .

B.4. For $\mathrm{k}=1$ to $\mathrm{np}$ do

B.4.1. Calculate and according to Eq. (5) and (6) respectively.

B.4.2. End do

B.5. Determine the set of disjoint paths. 
B.6. Construct the capacity vector X using Eq. (7).

B.7. Evaluate using $\mathrm{Eq}$ (3).

End.

Given $=(10,10)$ and $\mathrm{T}=11$, withthe consumed capacity shown in Table 2. Table 4,5 summarizes the values of, the set of disjoint paths and respectively.

Table 4. The disjoint MPS

\begin{tabular}{|l|l|}
\hline Disjoint Pair & Commonly-used arcs \\
\hline $\mathrm{MP}_{111} \cap \mathrm{MP}_{113}$ & $\varphi$ \\
\hline $\mathrm{MP}_{111} \cap \mathrm{MP}_{122}$ & $\varphi$ \\
\hline $\mathrm{MP}_{112} \cap \mathrm{MP}_{122}$ & $\varphi$ \\
\hline $\mathrm{MP}_{113} \cap \mathrm{MP}_{121}$ & $\varphi$ \\
\hline
\end{tabular}

Table 5. ${ }^{w j}$ Values

\begin{tabular}{|l|l|l|l|l|}
\hline $\mathbf{j}$ & $\mathbf{M P}_{\mathrm{ijk}}$ & $\mathbf{b}_{\mathbf{k}}{ }^{11}$ & $\mathbf{b}_{\mathbf{k}}{ }^{12}$ & $\mathbf{B}_{\mathbf{k}}$ \\
\hline 1 & $\left\{\mathrm{a}_{1}, \mathrm{a}_{5}\right\}$ & 2 & 3 & 8 \\
\hline 2 & $\left\{\mathrm{a}_{1}, \mathrm{a}_{6}, \mathrm{a}_{9}\right\}$ & 3 & 5 & 10 \\
\hline 3 & $\left\{\mathrm{a}_{2}, \mathrm{a}_{7}, \mathrm{a}_{9}\right\}$ & 2 & 4 & 10 \\
\hline 4 & $\left\{\mathrm{a}_{1}, \mathrm{a}_{6}, \mathrm{a}_{14}\right\}$ & 2 & 4 & 10 \\
\hline 5 & $\left\{\mathrm{a}_{2}, \mathrm{a}_{7}, \mathrm{a}_{14}\right\}$ & 2 & 3 & 10 \\
\hline
\end{tabular}

Finally, Table 6 summarizes the candidate vectors. The corresponding reliability is $=0.999988$. The reliability values for other sources and sinks are shown in Table 7.

Table 6. The candidate capacity vectors

\begin{tabular}{|l|l|l|}
\hline$\left(\mathbf{b}^{11}, \mathbf{b}^{12}\right)$ & \begin{tabular}{l}
$\left.\left(\mathbf{x}_{1}, \mathbf{x}_{2}, \mathbf{x}_{3}, \mathbf{x}_{4}, \mathbf{x}_{5}, \mathbf{x}_{6}, \mathbf{x}_{7}, \mathbf{x}_{8}, \mathbf{x}_{9}, \mathbf{x}_{10}, \mathbf{x}, \mathbf{x}_{12}, \mathbf{x}_{13}, \mathbf{x}_{14}\right)\right)$ \\
\hline$\left(\mathrm{b}_{1}{ }^{11}, \mathrm{~b}_{3}{ }^{12}\right)=(2,4)$
\end{tabular} & $\begin{array}{l}\text { QP } \\
\text { candidate }\end{array}$ \\
\hline$\left.\left(\mathrm{b}_{3}{ }^{11}, \mathrm{~b}_{1}{ }^{12}\right)=(2,3) 0,0,2,0,4,0,4,0,0,0,0,0\right)$ & $X_{1}$ \\
\hline$\left(\mathrm{b}_{1}{ }^{11}, \mathrm{~b}_{5}{ }^{12}\right)=(2,3)$ & $(3,2,0,0,3,0,2,0,2,0,0,0,0,0)$ & $X_{2}$ \\
\hline$\left(\mathrm{b}_{5}{ }^{11}, \mathrm{~b}_{1}{ }^{12}\right)=(2,3)$ & $(3,2,0,0,0,3,0,2,0,0,0,0,0,0,2)$ & $X_{4}$ \\
\hline$\left(\mathrm{b}_{5}{ }^{11}, \mathrm{~b}_{2}{ }^{12}\right)=(2,5)$ & $(5,2,0,0,0,5,2,0,5,0,0,0,0,2)$ & $X_{5}$ \\
\hline$\left(\mathrm{b}_{5}{ }^{11}, \mathrm{~b}_{5}{ }^{12}\right)=(3,3)$ & $(3,3,0,0,3,0,3,0,3,0,0,0,0,3)$ & $X_{6}$ \\
\hline$\left(\mathrm{b}_{4}{ }^{11}, \mathrm{~b}_{5}{ }^{12}\right)=(2,4)$ & $(2,4,0,0,0,2,4,0,4,0,0,0,0,2)$ & $X_{7}$ \\
\hline$\left(\mathrm{b}_{3}{ }^{11}, \mathrm{~b}_{4}{ }^{12}\right)=(2,4)$ & $(4,2,0,0,0,4,2,0,2,0,0,0,0,4)$ & $X_{8}$ \\
\hline
\end{tabular}

Table 7. Values of $R_{D^{2}, T}$ for different values of $\mathrm{d}_{\mathrm{wj}}$

\begin{tabular}{|c|c|c|}
\hline $\begin{array}{l}\text { Between different } \\
\text { sources and sinks }\end{array}$ & $\mathrm{MP}_{\mathrm{i}, \mathrm{j}, \mathrm{k}}$ & $R_{D_{w j}^{2}, T}$ \\
\hline$((1,2) \rightarrow 1)$ & $\begin{array}{l}\operatorname{MP}_{1,2,1}{ }_{1}=\left\{\mathrm{a}_{1}, \mathrm{a}_{5}\right\} \\
\mathrm{MP}_{1,2,1,2}=\left\{\mathrm{a}_{1}, \mathrm{a}_{6}, \mathrm{a}_{9}\right\} \\
\mathrm{MP}_{1,2,1}{ }^{\prime}=\left\{\mathrm{a}_{2}, \mathrm{a}_{7}, \mathrm{a}_{9}\right\} \\
\mathrm{MP}_{1,2,1,4}=\left\{\mathrm{a}_{3}, \mathrm{a}_{7}, \mathrm{a}_{9}\right\} \\
\operatorname{MP}_{1,2,1},_{5}=\left\{\mathrm{a}_{4}, \mathrm{a}_{8}, \mathrm{a}_{9}, \mathrm{a}_{13}\right\}\end{array}$ & 0.999990 \\
\hline$(2 \rightarrow(1,2))$ & $\begin{array}{l}\mathrm{MP}_{2,1,2^{\prime} 1}=\left\{\mathrm{a}_{3}, \mathrm{a}_{7}, \mathrm{a}_{9}\right\} \\
\mathrm{MP}_{2,1,2,2}=\left\{\mathrm{a}_{4}, \mathrm{a}_{8}, \mathrm{a}_{9}, \mathrm{a}_{13}\right\} \\
\mathrm{MP}_{2,1,2^{\prime}{ }_{3}}=\left\{\mathrm{a}_{3}, \mathrm{a}_{7}, \mathrm{a}_{14}\right\} \\
\mathrm{MP}_{2,1,2,4}=\left\{\mathrm{a}_{4}, \mathrm{a}_{8}, \mathrm{a}_{13}, \mathrm{a}_{14}\right\} \\
\mathrm{MP}_{2,1,2^{\prime}{ }_{5}}=\left\{\mathrm{a}_{4}, \mathrm{a}_{8}, \mathrm{a}_{10}\right\} \\
\mathrm{MP}_{2,1,2^{\prime} 6}=\left\{\mathrm{a}_{4}, \mathrm{a}_{11}, \mathrm{a}_{12}\right\}\end{array}$ & 0.999986 \\
\hline$((1,2) \rightarrow 2)$ & 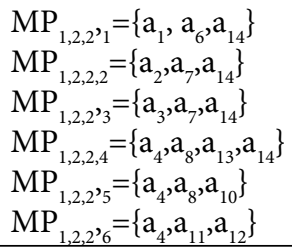 & 0.999995 \\
\hline
\end{tabular}

\section{Case C: Transmitting Demands Via Joint Paths from One Source to all Sinks}

We study how to calculates $R_{D_{\dot{y}}^{2}}, T$, the reliability from the source node $s_{1}$ to the sink node $t_{1}$ and $t_{2}$, where. There are five MPs from $s_{1}$ to both $t_{1}$ and $t_{2}$ shown in Table 8 and 9. In this case, the following constraint for each bandwidth should satisfy Eqs. (4), (5), (6) and

$$
\sum_{j=1}^{m} \sum_{i=1}^{u} \rho_{j} b_{j}^{i} \leq c\left(a^{*}\right)
$$

Table 8. The consumed capacity

\begin{tabular}{|l|l|l|l|l|l|}
\hline $\operatorname{Arc}$ & $\mathrm{W}^{11}\left(\mathrm{a}_{1}\right)$ & $\mathrm{W}^{12}\left(\mathrm{a}_{1}\right)$ & $\operatorname{Arc}$ & $\mathrm{W}^{11}\left(\mathrm{a}_{1}\right)$ & $\mathrm{W}^{12}\left(\mathrm{a}_{1}\right)$ \\
\hline $\mathrm{a}_{1}$ & 1 & 2 & $\mathrm{a}_{8}$ & 1 & 2 \\
\hline $\mathrm{a}_{2}$ & 1 & 2 & $\mathrm{a}_{9}$ & 1 & 2 \\
\hline $\mathrm{a}_{3}$ & 1 & 2 & $\mathrm{a}_{10}$ & 1 & 2 \\
\hline $\mathrm{a}_{4}$ & 1 & 2 & $\mathrm{a}_{11}$ & 1 & 2 \\
\hline $\mathrm{a}_{5}$ & 1 & 2 & $\mathrm{a}_{12}$ & 1 & 2 \\
\hline $\mathrm{a}_{6}$ & 1 & 2 & $\mathrm{a}_{13}$ & 1 & 2 \\
\hline $\mathrm{a}_{7}$ & 1 & 2 & $\mathrm{a}_{14}$ & 1 & 2 \\
\hline
\end{tabular}

Table 9. The data of each MP and $B_{k}$ values

\begin{tabular}{|l|l|l|l|}
\hline $\mathbf{k}$ & $\mathbf{M P}_{\mathrm{ijk}}$ & $\mathbf{L}\left(\mathbf{M P}_{\mathrm{ijk}}\right)$ & $\mathbf{B}_{\mathbf{k}}$ \\
\hline 1 & $\left\{\mathrm{a}_{1}, \mathrm{a}_{5}\right\}$ & 3 & 8 \\
\hline
\end{tabular}




\begin{tabular}{|l|l|l|l|}
\hline 2 & $\left\{\mathrm{a}_{1}, \mathrm{a}_{6}, \mathrm{a}_{9}\right\}$ & 7 & 10 \\
\hline 3 & $\left\{\mathrm{a}_{2}, \mathrm{a}_{7}, \mathrm{a}_{9}\right\}$ & 6 & 10 \\
\hline 4 & $\left\{\mathrm{a}_{1}, \mathrm{a}_{6}, \mathrm{a}_{14}\right\}$ & 6 & 10 \\
\hline 5 & $\left\{\mathrm{a}_{2}, \mathrm{a}_{7}, \mathrm{a}_{14}\right\}$ & 5 & 10 \\
\hline
\end{tabular}

where $\mathrm{a}^{*}$ is a commonly-used arc of two or more MPs .

Given $\mathrm{D}^{2}=(10,10)$ and $\mathrm{T}=11$, with the consumed capacity shown in Tables 10,11 summarizes the values of , the set of joint paths and respectively.

Table 10. The joint MPS

\begin{tabular}{|l|l|}
\hline Joint Pair & Commonly-used arcs $\left(\mathrm{a}^{\star}\right)$ \\
\hline $\mathrm{MP}_{111}, \mathrm{MP}_{112}, \mathrm{MP}_{121}$ & $\left\{\mathrm{a}_{1}\right\}$ \\
\hline $\mathrm{MP}_{112}, \mathrm{MP}_{113}$ & $\left\{\mathrm{a}_{9}\right\}$ \\
\hline $\mathrm{MP}_{112}, \mathrm{MP}_{121}$ & $\left\{\mathrm{a}_{6}\right\}$ \\
\hline $\mathrm{MP}_{113}, \mathrm{MP}_{122}$ & $\left\{\mathrm{a}_{7}\right\}$ \\
\hline $\mathrm{MP}_{121}, \mathrm{MP}_{122}$ & $\left\{\mathrm{a}_{14}\right\}$ \\
\hline
\end{tabular}

Table 11. $b_{k}^{w j}$ values

\begin{tabular}{|l|l|l|l|l|}
\hline$j$ & $\mathrm{MP}_{\mathrm{ijk}}$ & $\mathbf{b}_{\mathbf{j}}{ }^{1}$ & $\mathbf{b}_{\mathbf{j}}{ }^{2}$ & $\mathrm{C}\left(\mathbf{a}^{*}\right)$ \\
\hline 1 & $\left\{\mathrm{a}_{1}, \mathrm{a}_{5}\right\}$ & 2 & 3 & 8 \\
\hline 2 & $\left\{\mathrm{a}_{1}, \mathrm{a}_{6}, \mathrm{a}_{9}\right\}$ & 3 & 5 & 10 \\
\hline 3 & $\left\{\mathrm{a}_{2}, \mathrm{a}_{7}, \mathrm{a}_{9}\right\}$ & 2 & 4 & 10 \\
\hline 4 & $\left\{\mathrm{a}_{1}, \mathrm{a}_{6}, \mathrm{a}_{14}\right\}$ & 2 & 4 & 10 \\
\hline 5 & $\left\{\mathrm{a}_{2}, \mathrm{a}_{7}, \mathrm{a}_{14}\right\}$ & 2 & 3 & 10 \\
\hline
\end{tabular}

Finally, each $\mathrm{x}_{1}$ in the capacity vector $\mathrm{X}, \mathrm{X}=\left(\mathrm{x}_{1}, \mathrm{X}_{2}, \mathrm{X}_{3}\right.$ $\left., \mathrm{x}_{4}, \mathrm{x}_{5}, \mathrm{x}_{6}, \mathrm{x}_{7}, \mathrm{x}_{8}, \mathrm{x}_{9}, \mathrm{x}_{10}, \mathrm{x}_{11}, \mathrm{x}_{12}, \mathrm{x}_{13}, \mathrm{x}_{14}\right)$, is constructed by Eq. (7). Table 12 summarizes the candidate vectors, the corresponding $R_{D^{2}, 1}=0.999988$ the reliability value from s2 to both $\mathrm{t} 1$ and $\mathrm{t} 2$ shown in Table 13.

Table 12. The candidate capacity vectors

\begin{tabular}{|l|l|l|}
\hline$\left(\mathbf{b}^{11}, \mathbf{b}^{12}\right)$ & $\begin{array}{l}\left(\mathrm{x}_{1}, \mathrm{x}_{2}, \mathrm{x}_{3}, \mathrm{x}_{4}, \mathbf{x}_{5}, \mathrm{x}_{6}, \mathrm{x}_{7}, \mathbf{x}_{8}, \mathrm{x}_{9}, \mathrm{x}_{10}, \mathbf{x}\right. \\
\left.{ }_{11}, \mathbf{x}_{12} \mathbf{x}_{13}, \mathbf{x}_{14}\right)\end{array}$ & $\begin{array}{l}\mathrm{QP} \\
\text { candidate }\end{array}$ \\
\hline$\left(\mathrm{b}_{1}{ }^{11}, \mathrm{~b}_{2}{ }^{12}\right)=(2,5)$ & $(7,0,0,0,2,5,0,0,5,0,0,0,0,0)$ & $x_{1}$ \\
\hline$\left(\mathrm{b}_{2}{ }^{11}, \mathrm{~b}_{1}{ }^{12}\right)=(3,3)$ & $(6,0,0,0,3,3,0,0,3,0,0,0,0,0)$ & $x_{2}$ \\
\hline$\left(\mathrm{b}_{1}{ }^{11}, \mathrm{~b}_{4}{ }^{12}\right)=(2,4)$ & $(6,0,0,0,2,4,0,0,0,0,0,0,0,4)$ & $x_{3}$ \\
\hline
\end{tabular}

\begin{tabular}{|l|l|l|}
\hline$\left(\mathrm{b}_{4}{ }^{11}, \mathrm{~b}_{1}{ }^{12}\right)=(2,3)$ & $(5,0,0,0,3,2,0,0,0,0,0,0,0,2)$ & $x_{4}$ \\
\hline$\left(\mathrm{b}_{2}{ }^{11}, \mathrm{~b}^{12}\right)=(3,4)$ & $(3,4,0,0,0,3,4,0,7,0,0,0,0,0)$ & $x_{5}$ \\
\hline$\left(\mathrm{b}^{11}, \mathrm{~b}_{2}{ }^{12}\right)=(2,5)$ & $(5,2,0,0,0,5,2,0,7,0,0,0,0,0)$ & $x_{6}$ \\
\hline$\left(\mathrm{b}^{11}, \mathrm{~b}_{4}{ }^{12}\right)=(3,4)$ & $(7,0,0,0,0,7,0,0,3,0,0,0,0,4)$ & $x_{7}$ \\
\hline$\left(\mathrm{b}_{4}{ }^{11}, \mathrm{~b}_{2}{ }^{12}\right)=(2,5)$ & $(7,0,0,0,0,7,0,0,5,0,0,0,0,2)$ & $x_{8}$ \\
\hline$\left(\mathrm{b}_{3}{ }^{11}, \mathrm{~b}_{5}{ }^{12}\right)=(2,3)$ & $(0,5,0,0,0,0,5,0,2,0,0,0,0,3)$ & $x_{9}$ \\
\hline$\left(\mathrm{b}_{5}{ }^{11}, \mathrm{~b}_{3}{ }^{12}\right)=(2,4)$ & $(0,6,0,0,0,0,6,0,4,0,0,0,0,2)$ & $x_{10}$ \\
\hline$\left(\mathrm{b}_{4}{ }^{11}, \mathrm{~b}_{5}{ }^{12}\right)=(2,4)$ & $(2,3,0,0,0,2,3,0,0,0,0,0,0,5)$ & $x_{11}$ \\
\hline$\left(\mathrm{b}_{5}{ }^{11}, \mathrm{~b}_{4}{ }^{12}\right)=(2,4)$ & $(4,2,0,0,0,4,2,0,0,0,0,0,0,6)$ & $x_{12}$ \\
\hline
\end{tabular}

Table 13. Values of $R_{D^{2}, T}$ for different values of $\mathrm{d}_{\mathrm{wj}}$

\begin{tabular}{|l|l|l|l|}
\hline $\begin{array}{l}\text { Between } \\
\text { different } \\
\text { sources and } \\
\text { sinks }\end{array}$ & $\mathrm{MP}_{\mathrm{i}, \mathrm{j}, \mathrm{k}}$ & $R_{D_{w j}^{2}, T}$ & $\begin{array}{l}\text { The } \\
\text { value of } \\
R_{D_{w j}^{2}}{ }^{2}\end{array}$ \\
\hline$(2 \rightarrow(1,2))$ & $\begin{array}{l}\mathrm{MP}_{2,1,2,1}=\left\{\mathrm{a}_{4}, \mathrm{a}_{8}, \mathrm{a}_{9}, \mathrm{a}_{13}\right\} \\
\mathrm{MP}_{2,1,2_{2}}=\left\{\mathrm{a}_{3}, \mathrm{a}_{7}, \mathrm{a}_{14}\right\} \\
\mathrm{MP}_{2,1,2,3}=\left\{\mathrm{a}_{4}, \mathrm{a}_{8}, \mathrm{a}_{13}, \mathrm{a}_{14}\right\} \\
\mathrm{MP}_{2,1,2{ }^{2} 4}=\left\{\mathrm{a}_{4}, \mathrm{a}_{8}, \mathrm{a}_{10}\right\}\end{array}$ & $R_{D^{2}, 11}$ & 0.999968 \\
& $\mathrm{MP}_{2,1,2_{5}{ }_{5}=\left\{\mathrm{a}_{4}, \mathrm{a}_{11}, \mathrm{a}_{12}\right\}}$ & & \\
\hline
\end{tabular}

\section{Case D: Transmitting Multiple Demands Via Joint Paths from all Sources to all Sinks}

The system reliability $\mathrm{R}_{\mathrm{D}, \mathrm{T}}$ of the multi-source multi-sink flow network can be calculated by using the inclusionexclusion rule according to the generated set of all lower boundary points for $(\mathrm{D}, \mathrm{T})$. Given $\mathrm{D}^{4}=(5,5,5,5)$ and $\mathrm{T}$ $=11$, with the consumed capacity $\mathrm{W}^{11}\left(\mathrm{a}_{1}\right)=\mathrm{W}^{12}\left(\mathrm{a}_{1}\right)=1, \mathrm{~W}^{21}$ $\left(a_{1}\right)=W^{22}\left(a_{1}\right)=2$.. Table 14-16 summarizes the values of, the set of joint paths and, and the candidate capacity vectors respectively. The corresponding reliability $R_{D^{2}, 11}=0.999998$. In addition, using different consumed capacity $\mathrm{W}^{11}\left(\mathrm{a}_{1}\right)=\mathrm{W}^{12}\left(\mathrm{a}_{1}\right)=\mathrm{W}^{21}\left(\mathrm{a}_{1}\right)=\mathrm{W}^{22}\left(\mathrm{a}_{1}\right)=1$. Table 17-19 summarizes the values of, the set of joint paths and, and the candidate capacity vectors respectively. The corresponding reliability $R_{D^{2}, 11}=0.999998$. 
Table 14. The data of each MP and $B_{k}$ values

\begin{tabular}{|l|l|l|l|}
\hline $\mathrm{k}$ & $\mathrm{MP}_{\mathrm{ijk}}$ & $\mathrm{L}\left(\mathrm{MP}_{\mathrm{ijk}}\right)$ & $\mathrm{B}_{\mathrm{k}}$ \\
\hline 1 & $\left\{\mathrm{a}_{1}, \mathrm{a}_{5}\right\}$ & 3 & 8 \\
\hline 2 & $\left\{\mathrm{a}_{1}, \mathrm{a}_{6}, \mathrm{a}_{9}\right\}$ & 7 & 10 \\
\hline 3 & $\left\{\mathrm{a}_{2}, \mathrm{a}_{7}, \mathrm{a}_{9}\right\}$ & 6 & 10 \\
\hline 4 & $\left\{\mathrm{a}_{1}, \mathrm{a}_{6}, \mathrm{a}_{14}\right\}$ & 6 & 10 \\
\hline 5 & $\left\{\mathrm{a}_{2}, \mathrm{a}_{7}, \mathrm{a}_{14}\right\}$ & 5 & 10 \\
\hline 6 & $\left\{\mathrm{a}_{3}, \mathrm{a}_{7}, \mathrm{a}_{9}\right\}$ & 8 & 10 \\
\hline 7 & $\left\{\mathrm{a}_{3}, \mathrm{a}_{7}, \mathrm{a}_{14}\right\}$ & 7 & 10 \\
\hline 8 & $\left\{\mathrm{a}_{4}, \mathrm{a}_{8}, \mathrm{a}_{9}, \mathrm{a}_{13}\right\}$ & 8 & 12 \\
\hline 9 & $\left\{\mathrm{a}_{4}, \mathrm{a}_{8}, \mathrm{a}_{13}, \mathrm{a}_{14}\right\}$ & 7 & 12 \\
\hline 10 & $\left\{\mathrm{a}_{4}, \mathrm{a}_{8}, \mathrm{a}_{10}\right\}$ & 5 & 8 \\
\hline 11 & $\left\{\mathrm{a}_{4}, \mathrm{a}_{11}, \mathrm{a}_{12}\right\}$ & 7 & 10 \\
\hline
\end{tabular}

Table 15. $b_{k}$ wj values

\begin{tabular}{|l|l|l|l|l|l|}
\hline $\mathbf{j}$ & $\mathbf{M P}_{\mathrm{ijk}}$ & $\mathbf{b}_{\mathbf{j}}{ }^{11}$ & $\mathbf{b}_{\mathbf{j}}{ }^{12}$ & $\mathbf{b}_{\mathbf{j}}{ }^{21}$ & $\mathbf{b}_{\mathbf{j}}^{22}$ \\
\hline 1 & $\left\{\mathrm{a}_{1}, \mathrm{a}_{5}\right\}$ & 1 & 2 & 1 & 2 \\
\hline 2 & $\left\{\mathrm{a}_{1}, \mathrm{a}_{6}, \mathrm{a}_{9}\right\}$ & 2 & 3 & 2 & 3 \\
\hline 3 & $\left\{\mathrm{a}_{2}, \mathrm{a}_{7}, \mathrm{a}_{9}\right\}$ & 1 & 2 & 1 & 2 \\
\hline 4 & $\left\{\mathrm{a}_{1}, \mathrm{a}_{6}, \mathrm{a}_{14}\right\}$ & 1 & 2 & 1 & 2 \\
\hline 5 & $\left\{\mathrm{a}_{2}, \mathrm{a}_{7}, \mathrm{a}_{14}\right\}$ & 1 & 2 & 1 & 2 \\
\hline 6 & $\left\{\mathrm{a}_{3}, \mathrm{a}_{7}, \mathrm{a}_{9}\right\}$ & 2 & 4 & 2 & 4 \\
\hline 7 & $\left\{\mathrm{a}_{3}, \mathrm{a}_{7}, \mathrm{a}_{14}\right\}$ & 2 & 3 & 2 & 3 \\
\hline 8 & $\left\{\mathrm{a}_{4}, \mathrm{a}_{8}, \mathrm{a}_{9}, \mathrm{a}_{13}\right\}$ & 2 & 4 & 2 & 4 \\
\hline 9 & $\left\{\mathrm{a}_{4}, \mathrm{a}_{8}, \mathrm{a}_{13}, \mathrm{a}_{14}\right\}$ & 2 & 3 & 2 & 3 \\
\hline 10 & $\left\{\mathrm{a}_{4}, \mathrm{a}_{8}, \mathrm{a}_{10}\right\}$ & 1 & 2 & 1 & 2 \\
\hline 11 & $\left\{\mathrm{a}_{4}, \mathrm{a}_{11}, \mathrm{a}_{12}\right\}$ & 2 & 3 & 2 & 3 \\
\hline
\end{tabular}

Table 16. The candidate capacity vectors

\begin{tabular}{|l|l|l|}
\hline$\left(\mathbf{b}^{11}, \mathbf{b}^{12}, \mathbf{b}^{21}, \mathbf{b}^{22}\right)$ & $\begin{array}{l}\left(\mathbf{x}_{1}, \mathbf{x}_{2}, \mathbf{x}_{3}, \mathbf{x}_{4}, \mathbf{x}_{5}, \mathbf{x}_{6}, \mathbf{x}_{7}, \mathbf{x}_{8}, \mathbf{x}_{9}, \mathbf{x}_{10},\right. \\
\left.\mathbf{x}_{11}, \mathbf{x}_{12}, \mathbf{x}_{13}, \mathbf{x}_{14}\right)\end{array}$ & $\begin{array}{l}\mathbf{Q P} \\
\text { candidate }\end{array}$ \\
\hline a5 $\left\{\mathrm{a}_{1}, \mathrm{a}_{5}\right\}$ & $(7,0,0,0,2,5,0,0,5,0,0,0,0,0)$ & $x_{1}$ \\
\hline a10 $\left\{\mathrm{a}_{4}, \mathrm{a}_{8}, \mathrm{a}_{10}\right\}$ & $(6,0,0,0,3,3,0,0,3,0,0,0,0,0)$ & $x_{2}$ \\
\hline a11 $\left\{\mathrm{a}_{4}, \mathrm{a}_{11}, \mathrm{a}_{12}\right\}$ & $(6,0,0,0,2,4,0,0,0,0,0,0,0,4)$ & $x_{3}$ \\
\hline a12 $\left\{\mathrm{a}_{4}, \mathrm{a}_{11}, \mathrm{a}_{12}\right\}$ & $(5,0,0,0,3,2,0,0,0,0,0,0,0,2)$ & $x_{4}$ \\
\hline
\end{tabular}

Table 17. The data of each MP and $B_{k}$ values

\begin{tabular}{|l|l|l|l|}
\hline $\mathbf{k}$ & $\mathrm{MP}_{\mathrm{ijk}}$ & $\mathrm{L}\left(\mathrm{MP}_{\mathrm{ij} \mathbf{k}}\right)$ & $\mathbf{B}_{\mathbf{k}}$ \\
\hline 1 & $\left\{\mathrm{a}_{1}, \mathrm{a}_{5}\right\}$ & 3 & 8 \\
\hline 2 & $\left\{\mathrm{a}_{1}, \mathrm{a}_{6}, \mathrm{a}_{9}\right\}$ & 7 & 10 \\
\hline
\end{tabular}

\begin{tabular}{|l|l|l|l|}
\hline 3 & $\left\{\mathrm{a}_{2}, \mathrm{a}_{7}, \mathrm{a}_{9}\right\}$ & 6 & 10 \\
\hline 4 & $\left\{\mathrm{a}_{1}, \mathrm{a}_{6}, \mathrm{a}_{14}\right\}$ & 6 & 10 \\
\hline 5 & $\left\{\mathrm{a}_{2}, \mathrm{a}_{7}, \mathrm{a}_{14}\right\}$ & 5 & 10 \\
\hline 6 & $\left\{\mathrm{a}_{3}, \mathrm{a}_{7}, \mathrm{a}_{9}\right\}$ & 8 & 10 \\
\hline 7 & $\left\{\mathrm{a}_{3}, \mathrm{a}_{7}, \mathrm{a}_{14}\right\}$ & 7 & 10 \\
\hline 8 & $\left\{\mathrm{a}_{4}, \mathrm{a}_{8}, \mathrm{a}_{9}, \mathrm{a}_{13}\right\}$ & 8 & 12 \\
\hline 9 & $\left\{\mathrm{a}_{4}, \mathrm{a}_{8}, \mathrm{a}_{13}, \mathrm{a}_{14}\right\}$ & 7 & 12 \\
\hline 10 & $\left\{\mathrm{a}_{4}, \mathrm{a}_{8}, \mathrm{a}_{10}\right\}$ & 5 & 8 \\
\hline 11 & $\left\{\mathrm{a}_{4}, \mathrm{a}_{11}, \mathrm{a}_{12}\right\}$ & 7 & 10 \\
\hline
\end{tabular}

Table 18. $b_{k}{ }^{\text {wj }}$ values

\begin{tabular}{|l|l|l|l|l|l|}
\hline $\mathrm{j}$ & $\mathrm{MP}_{\mathrm{ijk}}$ & $\mathbf{b}_{\mathbf{j}}{ }^{\mathbf{1}}$ & $\mathbf{b}_{\mathrm{j}}{ }^{2}$ & $\mathbf{b}_{\mathbf{j}}{ }^{3}$ & $\mathbf{b}_{\mathbf{j}}{ }^{4}$ \\
\hline 1 & $\left\{\mathrm{a}_{1}, \mathrm{a}_{5}\right\}$ & 1 & 1 & 1 & 1 \\
\hline 2 & $\left\{\mathrm{a}_{1}, \mathrm{a}_{6}, \mathrm{a}_{9}\right\}$ & 2 & 2 & 2 & 2 \\
\hline 3 & $\left\{\mathrm{a}_{2}, \mathrm{a}_{7}, \mathrm{a}_{9}\right\}$ & 1 & 1 & 1 & 1 \\
\hline 4 & $\left\{\mathrm{a}_{1}, \mathrm{a}_{6}, \mathrm{a}_{14}\right\}$ & 1 & 1 & 1 & 1 \\
\hline 5 & $\left\{\mathrm{a}_{2}, \mathrm{a}_{7}, \mathrm{a}_{14}\right\}$ & 1 & 1 & 1 & 1 \\
\hline 6 & $\left\{\mathrm{a}_{3}, \mathrm{a}_{7}, \mathrm{a}_{9}\right\}$ & 2 & 2 & 2 & 2 \\
\hline 7 & $\left\{\mathrm{a}_{3}, \mathrm{a}_{7}, \mathrm{a}_{14}\right\}$ & 2 & 2 & 2 & 2 \\
\hline 8 & $\left\{\mathrm{a}_{4}, \mathrm{a}_{8}, \mathrm{a}_{9}, \mathrm{a}_{13}\right\}$ & 2 & 2 & 2 & 2 \\
\hline 9 & $\left\{\mathrm{a}_{4}, \mathrm{a}_{8}, \mathrm{a}_{13}, \mathrm{a}_{14}\right\}$ & 2 & 2 & 2 & 2 \\
\hline 10 & $\left\{\mathrm{a}_{4}, \mathrm{a}_{8}, \mathrm{a}_{10}\right\}$ & 1 & 1 & 1 & 1 \\
\hline 11 & $\left\{\mathrm{a}_{4}, \mathrm{a}_{11}, \mathrm{a}_{12}\right\}$ & 2 & 2 & 2 & 2 \\
\hline
\end{tabular}

Table 19. The candidate capacity vectors

\begin{tabular}{|l|l|l|}
\hline$\left(\mathbf{b}^{1}, \mathbf{b}^{2}, \mathbf{b}^{3}, \mathbf{b}^{4}\right)$ & $\begin{array}{l}\left(\mathbf{x}_{1}, \mathbf{x}_{2}, \mathbf{x}_{3}, \mathbf{x}_{4}, \mathbf{x}_{5}, \mathbf{x}_{6}, \mathbf{x}_{7}, \mathbf{x}_{8}, \mathbf{x}_{9}, \mathbf{x}_{10},\right. \\
\left.\mathbf{x}_{11}, \mathbf{x}_{12}, \mathbf{x}_{13}, \mathbf{x}_{14}\right)\end{array}$ & $\begin{array}{l}\text { QP } \\
\text { candidate }\end{array}$ \\
\hline $\begin{array}{l}\mathrm{a} 2\left\{\mathrm{a}_{2}, \mathrm{a}_{7}, \mathrm{a}_{9}\right\} \\
\left\{\mathrm{a}_{2}, \mathrm{a}_{7}, \mathrm{a}_{14}\right\}\end{array}$ & $(0,8,0,0,0,0,8,0,4,0,0,0,0,4)$ & $x_{1}$ \\
\hline $\mathrm{a} 5\left\{\mathrm{a}_{1}, \mathrm{a}_{5}\right\}$ & $(0,4,0,0,4,0,0,0,0,0,0,0,0,0)$ & $x_{2}$ \\
\hline $\mathrm{a} 10\left\{\mathrm{a}_{4}, \mathrm{a}_{8}, \mathrm{a}_{10}\right\}$ & $(0,0,0,4,0,0,0,4,0,4,0,0,0,0)$ & $x_{3}$ \\
\hline $\mathrm{a} 11\left\{\mathrm{a}_{4}, \mathrm{a}_{11}, \mathrm{a}_{12}\right\}$ & $(0,0,0,8,0,0,0,0,0,0,8,8,0,0)$ & $x_{4}$ \\
\hline $\mathrm{a} 12\left\{\mathrm{a}_{4}, \mathrm{a}_{11}, \mathrm{a}_{12}\right\}$ & $(0,0,0,8,0,0,0,0,0,0,8,8,0,0)$ & $x_{5}$ \\
\hline $\begin{array}{l}\mathrm{a} 2\left\{\mathrm{a}_{2}, \mathrm{a}_{7}, \mathrm{a}_{9}\right\} \text { and } \\
\left\{\mathrm{a}_{2}, \mathrm{a}_{7}, \mathrm{a}_{14}\right\}\end{array}$ & $(0,8,0,0,0,0,8,0,4,0,0,0,0,4)$ & $x_{6}$ \\
\hline
\end{tabular}

\section{Conclusions}

The study is successfully evaluated the reliability in multisource multi-sink stochastic flow networks in different 
situations. Given the network information (arcs capacities, probabilities, lead times), required demand single or multiple based on the source and sink nodes, and the time constraints T. Situation A, transmitting with no restriction on determining the relationship between the group of paths, joint or disjoint. Situation B, transmitting the required demands via disjoint paths situation $\mathrm{C}$, transmitting demands through a group of joint paths, share one or more common arc. Finally, the general case when all sources send the required demands to the sink nodes via joint paths, evaluating, of a multi-source multi-sink flow network.

\section{References}

1. Chen YL, Chin YH. The quickest path problem, CoMPuters \& Operations Research. 1990; 12(7):153-61. https://doi. org/10.1016/0305-0548(90)90039-A.

2. Yeh WC, Lin LE, Chou YC, Chen YC. Optimal routing for multi-commodity in multistate flow network with time constraints, Quality Technology and Quantitative Management. 2013; 10(2):161-77. https://doi.org/10.1080 /16843703.2013.11673314.

3. Yung-Chen Hung, Gen-Huey Chen. Distributed algorithms for the quickest path problem, Parallel Computing. 1992; 18(7):823-34. https://doi. org/10.1016/0167-8191(92)90048-C

4. Martins EDV, dos Santos JLE. An algorithm for the quickest path problem, Operations Research Letters. 1997; 195-98. https://doi.org/10.1016/S0167-6377(97)00008-4.

5. Yi-Kuei Lin. Extend the quickest path problem to the system reliability evaluation for a stochastic-flow network. Computers and Operations Research, 30, 2003, pp. 567575. https://doi.org/10.1016/S0305-0548(02)00025-4.

6. Chen YL, Chin YH. The quickest path problem, Computers and Operations Research. 1990; 17:153-61. https://doi. org/10.1016/0305-0548(90)90039-A.

7. Yi-Kuei Lin. Time version of the shortest path problem in stochastic-flow network, Journal of Computational and Applied Mathematics. 2009; 228:150-57. https://doi. org/10.1016/j.cam.2008.09.003.

8. Yi-Kuei Lin. Optimal routing policy of a stochastic-flow network, Computers and Industrial Engineering. 2009; 56:1414-18. https://doi.org/10.1016/j.cie.2008.09.002.

9. Yi-Kuei Lin. Routing policy of stochastic-flow networks under time threshold and budget constraint, Expert Systems with Applications. 2009; 36:6076-81. https://doi. org/10.1016/j.eswa.2008.07.080.
10. Lin YK. Reliability of $\mathrm{k}$ separate minimal paths under both time and budget constraints, IEEE Transactions on Reliability. 2010; 59(1):183-90. https://doi.org/10.1109/ TR.2009.2035794.

11. Lin YK. Network reliability of a time-based multistate network under spare routing with $\rho$ minimal paths, IEEE Transactions on Reliability. 2011; 60(1):61-69. https://doi. org/10.1109/TR.2010.2103594.

12. Lin YK. Stochastic flow networks via multiple paths under time threshold and budget constraint, Computers and Mathematics with Applications. 2011; 62(6):2629-38. https://doi.org/10.1016/j.camwa.2011.08.002.

13. Chung-Chi Hsieh, Ming-Hsien Lin. Reliability-oriented multi-resource allocation in a stochastic-flow network, Reliability Engineering and System Safety. 2003; 81:155-61. https://doi.org/10.1016/S0951-8320(03)00082-6.

14. Qiang Liu, Hailin Zhang Xiaoxian Ma, Qingzhen Zhao. Genetic algorithm-based study on flow allocation in a multicommodity stochastic-flow network with unreliable nodes. Eighth ACIS International Conference on Software Engineering, Artificial Intelligence, Networking, and Parallel/Distributed Computing; 2007. p. 576-81. https:// doi.org/10.1109/SNPD.2007.261.

15. Liu Q, Zhao Q, Zang W. Study on multi-objective optimization of flow allocation in a multi-commodity stochastic-flow network with unreliable nodes, Journal of Applied Mathematics Computing. 2008; 28:185-98. https:// doi.org/10.1007/s12190-008-0093-9.

16. Hassan MR. Solving Flow Allocation Problems and Optimizing System Reliability of Multisource Multisink Stochastic Flow Network, The International Arab Journal of Information Technology. 2016; 13(5):477-83.

17. Janan X. On multistate system analysis, IEEE Transactions on Reliability. 1985; 34(4):329-37. https://doi.org/10.1109/ TR.1985.5222178.

18. Locks MO. Recursive disjoint products, inclusion-exclusion, and min-cut approximations, IEEE Transactions on Reliability. 1980; 29(5):425-43. https://doi.org/10.1109/ TR.1980.5220885.

19. Alexopoulos C. A Note on state-space decomposition methods for analyzing stochastic flow networks, IEEE Transactions on Reliability. 1995; 44:354-57. https://doi. org/10.1109/24.387394.

20. Zuo MJ, Tian Z, Huang HZ. An efficient method for reliability evaluation of multistate networks given all minimal path vectors, IIE Transactions. 2007; 39:811-17. https:// doi.org/10.1080/07408170601013653. 